\title{
Questões metodológicas envolvidas no design de experimentos de processamento de leitura com o uso do software PsychoPy
}

Methodological issues involved in the design of reading processing experiments using PsychoPy

\author{
Bernardo Kolling Limberger \\ Ana Paula Correa da Silva Biasibetti*
}

RESUMO: Este artigo tem como principal objetivo analisar o uso do programa PsychoPy na investigação do processamento de leitura no que tange ao desenvolvimento, execução e tratamento dos resultados de dois experimentos psicolinguísticos. Para tanto, o passo-apasso da montagem de dois testes de leitura, a saber, o teste Stroop e de uma tarefa de decisão lexical, será apresentado. Além disso, os pontos fortes e fracos do programa serão salientados. Entende-se que este estudo é relevante no sentido de que abarca o desafio metodológico da pesquisa experimental em relação ao grau de dificuldade encontrado na elaboração e na aplicação dos experimentos. Desse modo, esperamos contribuir para a divulgação dos recursos disponibilizados pelo PsychoPy - um programa de livre acesso, gratuito e de fácil utilização - à pesquisa brasileira.

PALAVRAS-CHAVE: Processamento de leitura. Métodos. PsychoPy.

\begin{abstract}
The main objective of this article is to analyze the use of PsychoPy in the investigation of reading processing by focusing on the design, implementation and handling of the results of two psycholinguistic experiments. To this end, it provides the step-by-step assembly procedures of the Stroop test and a lexical decision task. In addition, it highlights the strengths and weaknesses of the program. This study is relevant because it covers the methodological challenges of experimental research as to the degree of difficulty in designing and applying experiments. It is expected to contribute to the dissemination of the resources provided by PsychoPy-a free, opencode, user-friendly software-to the Brazilian research community.
\end{abstract}

KEYWORDS: Reading processing. Methods. PsychoPy.

\footnotetext{
* Doutor em Letras pela Pontifícia Universidade Católica do Rio Grande do Sul, professor da Universidade Federal de Pelotas. limberger.bernardo@gmail.com

** Doutora em Letras pela Pontifícia Universidade Católica do Rio Grande do Sul. biasibetti.ana@gmail.com.
} 


\section{Introdução}

Observa-se, no cenário atual da pesquisa linguística brasileira, um número relativamente reduzido de estudos que contemplam a questão experimental, em comparação à linguística teórica ou à aplicada. Os altos custos de aquisição e licenciamento dos softwares que possibilitam a criação e aplicação de testes psicométricos e psicolinguísticos são alguns dos aspectos que dificultam a realização dessas investigações. Além disso, o pouco conhecimento em programação - caso que se aplica à maior parte dos linguistas - torna a construção e implementação de experimentos ainda mais desafiadora e metodologicamente arriscada.

Sampaio (2017) salienta que, dentre os softwares voltados para a construção de experimentos que necessitam de pouco ou nenhum conhecimento sobre programação, os programas e-Prime (Psychology Software Tools, 2016) e PsychoPy (PEIRCE, 2007; 2009) são aqueles mais difundidos entre os pesquisadores.

Ambos os programas apresentam alta qualidade técnica e índices de confiabilidade elevados. As principais desvantagens do e-Prime, no entanto, dizem respeito ao seu custo de aquisição e licenciamento (elevado para a realidade das instituições brasileiras) e à impossibilidade de inovar na construção dos experimentos, haja vista que seu código fechado permite que apenas procedimentos pré-definidos sejam utilizados na construção dos experimentos. O PsychoPy, por outro lado, apresenta como principal vantagem o fato de ser um software gratuito e de código aberto, o que permite diversas adaptações e ajustes. Além disso, o tempo de aprendizagem necessário para manipulá-lo é considerado relativamente baixo, uma vez que a interface do programa é bastante intuitiva e as eventuais dificuldades podem ser facilmente sanadas através de fóruns on-line.

No entanto, apesar das vantagens elencadas, o levantamento realizado no buscador Google (em agosto de 2018) para as palavras-chave "PsychoPy", "linguística” e "português" encontrou apenas 458 resultados. No que diz respeito aos estudos 
brasileiros que fizeram uso do PsychoPy em suas etapas experimentais, Santos (2016), Rocha e Raso (2017), Souza Filho et al. (2017), Godoy et al. (2017), Gomes (2017), Oliveira (2018), Limberger (2018) e Biasibetti (2018) são alguns dos trabalhos mais recentes. Revisamos esses estudos a seguir, já exemplificando, brevemente, algumas das potencialidades do programa.

No primeiro estudo mencionado, conduzido por Santos (2016), partiu-se da hipótese de que a leitura e a audição simultâneas facilitariam a compreensão oral em língua estrangeira. Em síntese, a autora desenvolveu e aplicou um teste de reconhecimento de palavras faladas utilizando o PsychoPy para apresentar os estímulos sonoros e registrar os tempos de reação e acurácia.

Quanto à interação entre pistas prosódicas e pragmáticas para a interpretação ilocucionária de um enunciado, Rocha e Raso (2017) criaram um teste de percepção em que duas versões de um vídeo eram apresentadas, a saber, uma com a ilocução original e outra com uma ilocução alternativa. Os vídeos foram exibidos em ordem aleatória e o grau de adequação do enunciado ao contexto foi avaliado através de uma escala de classificação (rating scale) com notas de 1 a 5, sendo 1 completamente inadequado e 5 completamente adequado.

Souza Filho et al. (2017) investigaram o efeito das relações de coerência sobre o processamento pronominal anafórico através de um experimento de leitura autocadenciada. Para tanto, 48 frases foram apresentadas individualmente à medida que os participantes pressionavam uma determinada tecla. Após a leitura de cada frase, os participantes respondiam sim ou não a uma questão de compreensão. Também se utilizando dessa técnica de leitura, Godoy et al. (2017) testaram se o conhecimento de eventos enquanto pista pragmática afeta o processamento de sentenças isoladas. Para o experimento que avaliou o tempo de leitura, 32 sentenças formadas por um contexto preditivo foram lidas pelos participantes, seguidas da resposta sim ou não a uma questão relacionada à sentença. 
O processamento de pronomes-objeto em construções dativas por bilíngues falantes de português e inglês através da técnica de leitura automonitorada foi investigado por Gomes (2017). O experimento apresentava aleatoriamente 45 frases segmentadas palavra por palavra na tela do computador. $\mathrm{O}$ tempo de reação para a leitura dos estímulos apresentados foi registrado e, ao final da leitura de cada sentença, o participante respondia a uma pergunta-controle.

Oliveira (2018) analisou a percepção das formas pronominais clíticas que atuam na referência à segunda pessoa do singular em posição de complemento. Os estímulos referentes a 30 enunciados experimentais foram apresentados aleatoriamente e os participantes solicitados a responder uma questão interpretativa para cada enunciado.

Limberger (2018) trabalhou principalmente com estímulos linguísticos visuais para investigar influências interlinguísticas no processamento da leitura de participantes multilíngues falantes de português, hunsriqueano e alemão standard. Para tanto, um teste Stroop foi aplicado a fim de caracterizar a amostra e parear os grupos. Outros três experimentos foram aplicados com o objetivo de contemplar subcomponentes da leitura multilíngue: tarefa de consciência fonêmica, tarefa de decisão lexical e tarefa de compreensão de sentenças. As variáveis dependentes avaliadas no estudo e mensuradas pelo PsychoPy foram tempo de resposta e acurácia.

Por fim, Biasibetti (2018) tratou da percepção fonética das fricativas sibilantes não-vozeadas das variedades porto-alegrense e florianopolitana através dos testes de similaridade e de discriminação acelerada do tipo AX. Ambas as tarefas incluíram 108 pares de estímulos sonoros que foram apresentados aleatoriamente e classificados através de uma escala de Likert (teste de similaridade) de cinco pontos e dos rótulos idêntico/diferente (teste de discriminação). Além disso, a autora investigou a percepção da variação sociofonética das variantes sibilantes alveolar e palato-alveolar em coda silábica a partir de associações implícitas entre as referidas fricativas e o estereótipo de morador nativo através do Teste de Associação Implícita. 
Os estudos linguísticos aqui elencados revelam uma variedade de métodos experimentais que podem ser implementados no PsychoPy de forma relativamente simples e rápida, em comparação com outros softwares. Além disso, os estudos revisados mostram que é possível apresentar diversos tipos de estímulos (sonoros, visuais, audiovisuais etc.), investigar diversos níveis linguísticos (fonético, lexical, sintático e pragmático) e obter diversos tipos de resposta (escala, tempo de resposta, acurácia).

Tendo por objetivo principal analisar o uso do programa PsychoPy na investigação do processamento de leitura no que tange ao desenvolvimento, execução e tratamento dos resultados experimentos psicolinguísticos, apresentamos o teste Stroop e uma tarefa de decisão lexical na sequência. Essa exposição será seguida de uma discussão sobre o programa em relação aos experimentos apresentados. Esperamos mostrar aos pesquisadores da área e a outros interessados as principais vantagens e desvantagens do programa em termos de programação, execução e tratamento dos resultados.

\section{PsychoPy}

O PsychoPy (PEIRCE, 2007; 2009) é um programa gratuito voltado para a construção e aplicação de experimentos nas áreas de psicologia, neurociência e psicofísica com vistas à apresentação de estímulos e à coleta de dados psicométricos, tais como tempos de resposta/reação $(R T)$ e acurácia (accuracy). Assim sendo, o programa constitui uma importante ferramenta metodológica para a pesquisa linguística, pois psicolinguistas e foneticistas, entre outros, estão cada vez mais empenhados em investigar experimentalmente os diversos aspectos envolvidos na produção, na percepção e no processamento da linguagem, nos seus diversos níveis e em diferentes populações. 
Como alternativa a programas proprietários e, portanto, de códigos fechados, tal como o e-Prime (Psychology Software Tools Inc.), o PsychoPy conta com a colaboração de inúmeros usuários, além de tutoriais, templates e fóruns, que propagam de forma livre e gratuita as mais diversas soluções e alternativas para a construção de experimentos científicos. Assim sendo, o PsychoPy é altamente transparente no sentido de que programadores e outros pesquisadores têm total acesso ao programa, a fim de atestar sua confiabilidade, assim como há condições para identificar e corrigir rapidamente quaisquer erros.

Dentre as diversas funcionalidades do Psychopy, destaca-se a possibilidade de execução do programa nos ambientes operacionais Windows, MacOS e Linux. Também merece destaque o fato de que os experimentos podem ser executados localmente ou online ${ }^{1}$. Além disso, o experimento pode ser executado em um número virtualmente infinito de computadores sem a necessidade de licenças de uso, ou seja, é possível aplicar o teste simultaneamente em $n$ computadores apenas instalando o programa nesses equipamentos. Destacamos que, para que o hardware não influencie negativamente a captura dos dados, é necessário que o computador possua um cartão de processador gráfico que suporte OpenGL. Finalmente, sobressai-se o fato de que é possível acoplar o PsychoPy à ressonância magnética funcional, ao eletroencefalograma e ao equipamento de rastreamento ocular.

Desenvolvido integralmente em linguagem de programação Python, o PsychoPy permite que diversos tipos de experimentos sejam criados a partir da programação de scripts diretamente em Python no módulo Coder (cf. Figura 1) ou a partir de comandos simplificados no módulo Builder (cf. Figura 2).

\footnotetext{
${ }^{1}$ A interface para execução de experimentos online já está disponível na versão mais recente do programa (versão 3.0.0), lançada em julho de 2018. Disponível em: https://github.com/psychopy/psychopy/releases. Acesso em: 28 ago. 2018.
} 
Figura 1 - Módulo Coder do PsychoPy.

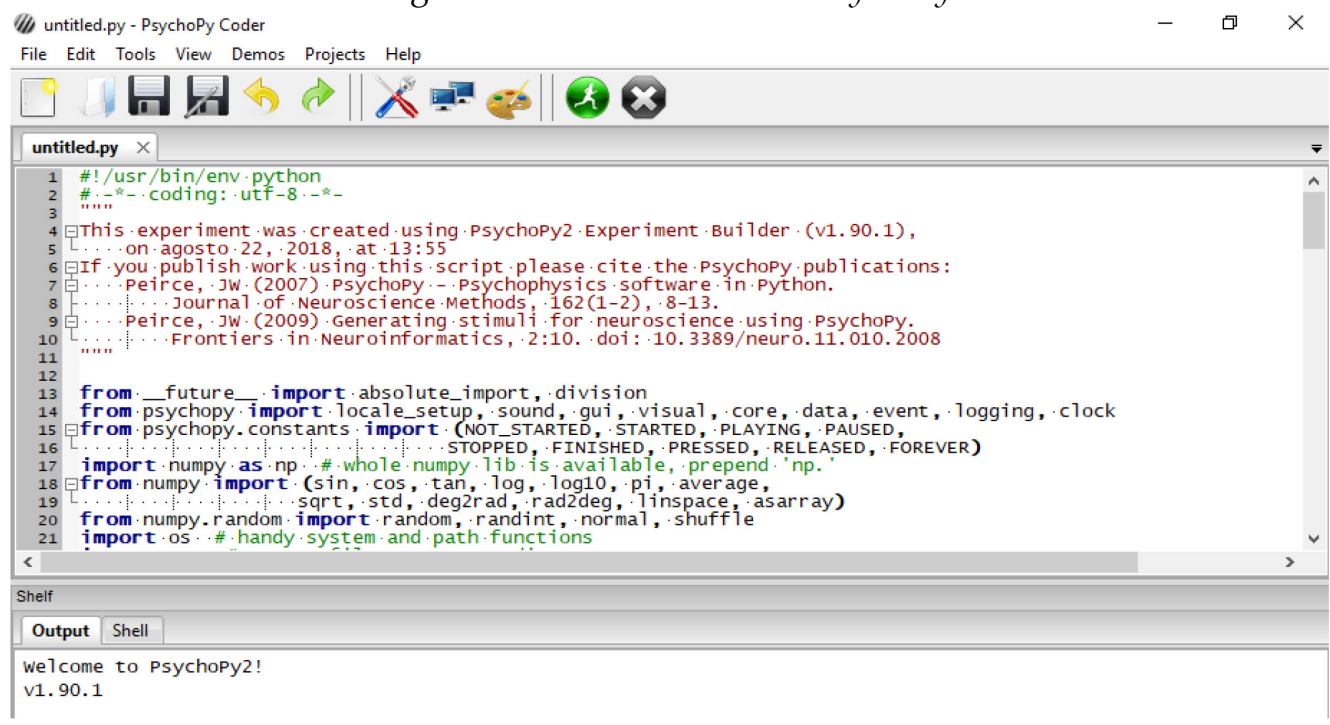

Fonte: Print screen do Coder do programa PsychoPy (PIERCE, 2007; 2009)

Figura 2 - Módulo Builder do PsychoPy.

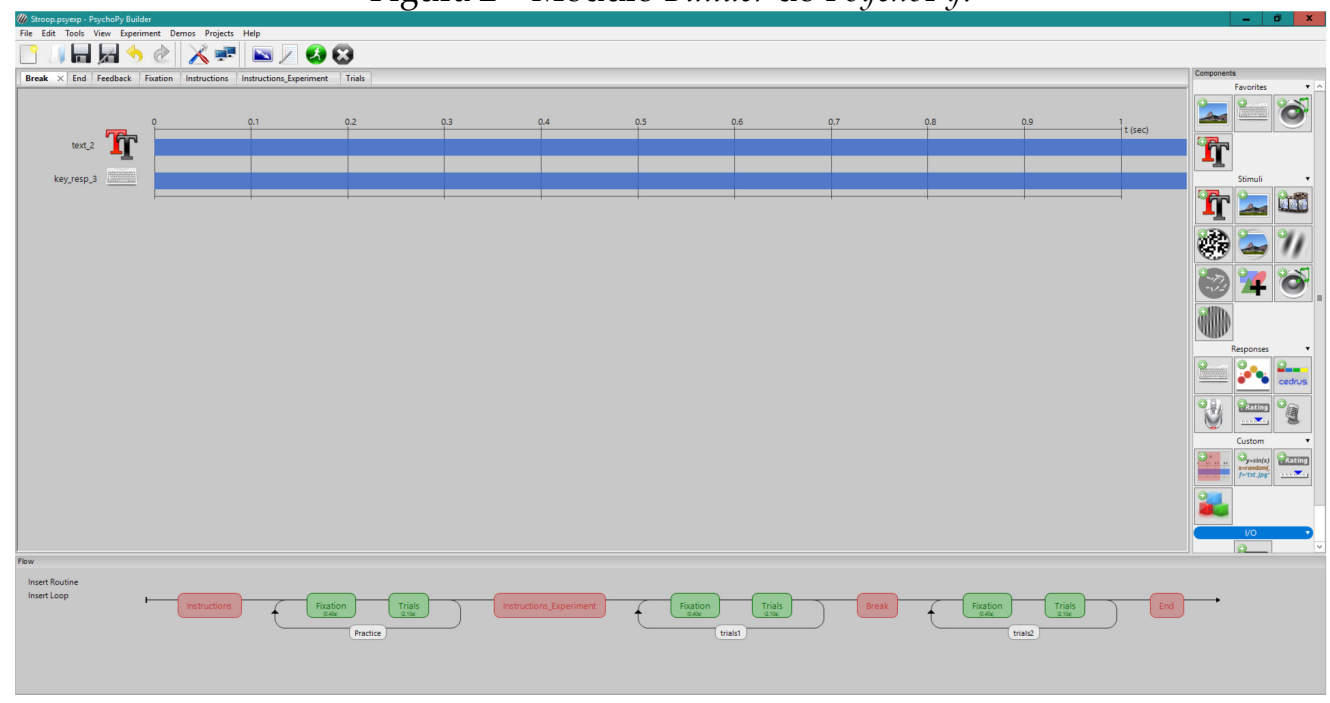

Fonte: Print screen do Builder do teste Stroop no programa PsychoPy (PIERCE, 2007; 2009).

Todos os procedimentos descritos deste ponto em diante dizem respeito ao módulo Builder. Funcional e intuitivo, o referido módulo propicia a rápida aprendizagem do PsychoPy.

Os estímulos, elementos gráficos e o método de resposta que serão inseridos no experimento como parte de sua estrutura (visível ou não aos participantes) são chamados de componentes. Os componentes, por sua vez, são inseridos dentro de rotinas que constituem as diferentes etapas de experimentação, tais como a instrução 
do teste, o treino, o teste em si, o feedback e a mensagem de finalização do teste, por exemplo. Componentes e rotinas são facilmente visualizados no módulo Builder: a tela superior exibe as rotinas com sua duração ao longo de uma linha do tempo, enquanto a tela inferior exibe as rotinas em um fluxograma (cf. Figura 2).

Em relação aos componentes do PsychoPy, há estímulos (stimuli), respostas (responses), customização (custom) e entrada/saída (Input/Output) que podem ser configurados em relação à duração de sua ativação.

A começar pelos estímulos, o PsychoPy permite a inserção simples e rápida de estímulos sonoros e visuais (imagens e vídeos) cuja exibição pode ser configurada nos modos aleatório ou fixo. Os estímulos sonoros podem ser arquivos do tipo mid, wav e ogg. As imagens, por sua vez, podem ser arquivos dos formatos tif, jpg, bmp e png. Os vídeos, por fim, podem ser arquivos mpeg, avi e mov. Os estímulos e outros elementos textuais que fazem parte do experimento podem ser inseridos através da planilha de dados ou diretamente no PsychoPy através da digitação do texto em campos específicos. Em princípio, o software não limita a extensão dos estímulos. Porém, os estímulos devem caber na tela, de modo que fiquem legíveis para os participantes.

O programa também permite que o pesquisador selecione diferentes possibilidades de resposta aos estímulos. É possível que o participante responda com o teclado (digitando uma resposta ou pressionando uma determinada tecla), com o microfone (gravando suas respostas vocalizadas), com um Button box (pressionando os botões de um console específico voltado para a pesquisa) ou com o mouse (clicando em algum ponto específico do ambiente de experimentação, tais como os pontos de uma escala de Likert, por exemplo). A exemplo, Biasibetti (2018) criou uma escala de Likert de cinco pontos para mensurar o grau de semelhança fonética entre as fricativas alveolar e palato-alveolar em onset e em coda silábica. O primeiro ponto corresponde à avaliação de que os dois estímulos sonoros são percebidos como muito semelhantes ao passo que o último ponto corresponde à percepção de que os estímulos são muito 
diferentes. Os participantes utilizaram o mouse para clicar em um dos cinco pontos e, com isso, registrarem sua resposta.

Diferentemente do programa E-Prime, a relação de estímulos que constituem o teste e as respostas ao teste, ou seja, suas condições experimentais, devem ser inseridas em uma planilha de dados salva na extensão .xlsx. Isso significa que o arquivo de condições do experimento deve ser construído em um editor de planilhas à parte do PsychoPy, tal como Excel, por exemplo. Essa planilha deve ser salva na mesma pasta em que estarão armazenados os estímulos que constarão no teste.

Ainda em relação aos componentes disponibilizados pelo PsychoPy no módulo Builder, há também opções customizadas, como a possibilidade de inserir códigos específicos de Python para a inclusão de uma rotina de feedback no treino de determinada tarefa. Outra alternativa é inserir um período no qual a tela fica estática ("Static"), por exemplo, quando se precisa de uma pausa antes da apresentação de determinados estímulos. Por fim, o componente de entrada/saída (I/O) permite que outros equipamentos (hardware) sejam incorporados ao PsychoPy, tais como o equipamento para rastreamento ocular.

Ao final da aplicação do teste, as informações obtidas para cada participante sobre seus tempos de resposta/reação, a acurácia, as respostas atribuídas e a ordem de execução dos estímulos, entre outros, são salvos na subpasta data automaticamente gerada pelo programa em três formatos de arquivo: (1) log files - apresenta o output cronológico de vários dados; (2) psydat - arquivo para análise em Python; e (3) arquivo com extensão .csv (comma separated value), para análise no programa estatístico R e/ou conversão no Excel.

Apresentamos na seção a seguir o passo-a-passo da construção dos experimentos desenvolvidos e aplicados por Limberger (2018) em estudo aprovado pelo Comitê de Ética em Pesquisa (CEP) da Pontifícia Universidade Católica do Rio Grande do Sul (PUCRS) em 23 de março de 2016. Antes da aplicação dos experimentos, 
os participantes leram com atenção e assinaram um Termo de Consentimento Livre e Esclarecido (TCLE).

O teste Stroop e a tarefa de decisão lexical apresentados a seguir foram programados na versão 1.85.23 do PsychoPy em um laptop HP Pavilion 14' com monitor com taxa de atualização de $59 \mathrm{~Hz}$ e resolução de tela de 1366 x 768 .

\section{Experimento 1: Teste Stroop}

O experimento denominado Stroop, desenvolvido pelo pesquisador homônimo em 1935, visa simular a habilidade desempenhada pelo controle inibitório, na qual é requerida a inibição deliberada de estímulos irrelevantes ou automáticos para a realização de determinada tarefa (MIYAKE et al., 2000). Limberger (2018) mensurou esse construto cognitivo nos diferentes grupos de participantes, pareando-os em função do controle inibitório, o qual tende a influenciar o desempenho de bilíngues e multilíngues em tarefas cognitivas.

O teste Stroop aqui descrito exigia dos participantes o foco na cor da palavra apresentada independentemente do texto exibido. As palavras VERDE, AZUL e ROSA (Figura 3) foram escolhidas devido à extensão semelhante por elas apresentada. Os participantes liam na tela do computador uma palavra por vez e eram solicitados a pressionar a tecla do computador que continha a etiqueta da cor em que a palavra havia sido exibida, inibindo, quando necessário, o texto. As cores utilizadas foram o verde, o azul e o rosa, sendo que a tecla " $\mathrm{j}$ " continha a etiqueta na cor verde, a tecla " 1 " continha a etiqueta na cor azul e a tecla "i", a etiqueta na cor rosa. As referidas teclas foram escolhidas devido a sua proximidade no teclado do computador.

Figura 3 - Representação esquemática do teste Stroop de cores. 


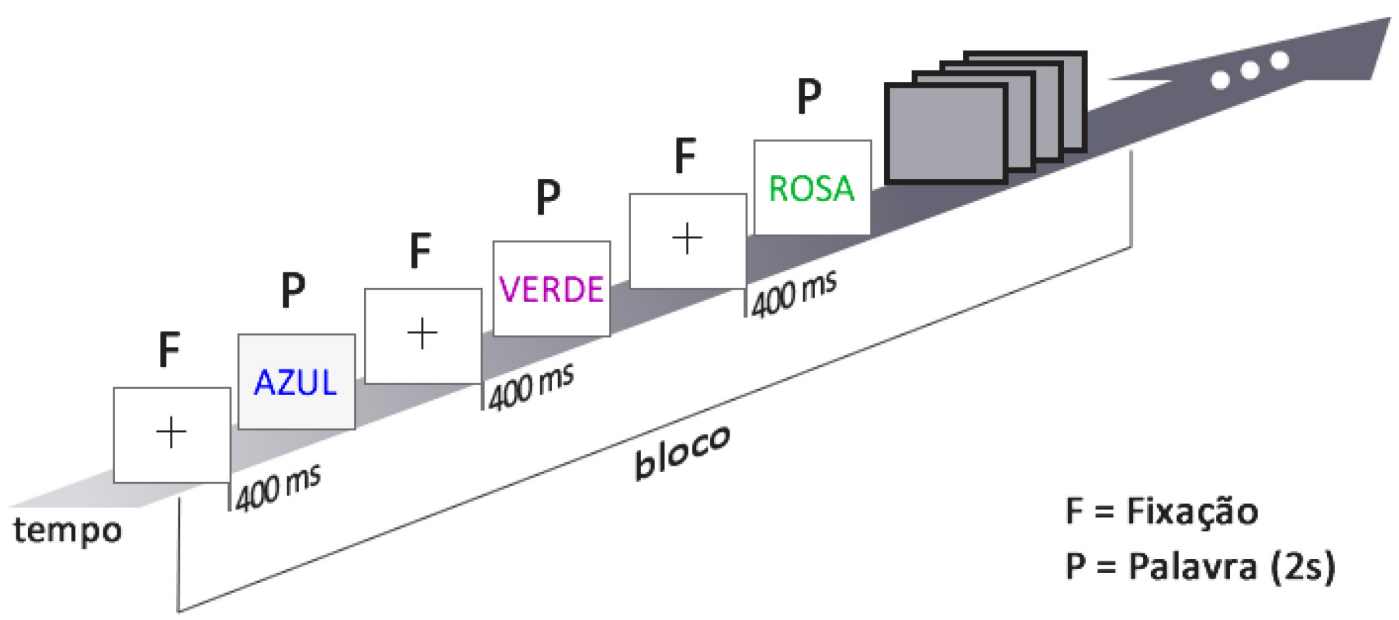

Fonte: os autores; imagem gerada no software Adobe Fireworks.

Os participantes eram confrontados com itens congruentes - nos quais a cor da palavra correspondia com o texto - e itens incongruentes - nos quais não havia congruência entre cor e texto. Nesses itens, o participante necessitava inibir ou substituir a tendência de fornecer a resposta mais dominante ou automática, isto é, a leitura do texto. Assim, além de possibilitar a avaliação do controle inibitório no processamento da leitura, pode-se avaliar a automaticidade dos processos de decodificação.

Primeiramente, os participantes realizaram um treino com oito estímulos e, em seguida, o teste com 60 estímulos. A palavra aparecia durante 2000 milissegundos (ms), seguida de um ponto de fixação (400 ms). Calcula-se a diferença entre os resultados nos itens incongruentes e congruentes, diferença essa denominada Efeito Stroop.

A seguir, expomos os passos da construção do experimento, a fim de exemplificar como ele é construído e facilitar a replicabilidade do uso do PsychoPy.

\subsection{Construção do experimento no PsychoPy}

Primeiramente, usamos o programa Excel para montar a planilha de condições com a relação de estímulos e respostas que se aplicam ao experimento (Figura 4). Os títulos das colunas na planilha não devem conter espaços, caracteres especiais ou 
diacríticos. Por sua vez, as palavras (ou frases ou textos) que constituem os estímulos textuais do experimento podem conter diacríticos. Cada linha corresponde a uma única pergunta ("trial”) do experimento. $\mathrm{O}$ arquivo de condições deve ser salvo com a extensão .xlsx na mesma pasta em que o experimento será salvo.

Figura 4 - Arquivo de condições do teste Stroop.

\begin{tabular}{|c|c|c|c|c|}
\hline & A & B & C & D \\
\hline 1 & word & colour & condition & correct \\
\hline 2 & ROSA & magenta & congruente & i \\
\hline 3 & ROSA & green & incongruente & j \\
\hline 4 & VERDE & green & congruente & j \\
\hline 5 & VERDE & blue & incongruente & l \\
\hline 6 & AZUL & blue & congruente & I \\
\hline 7 & AZUL & magenta & incongruente & i \\
\hline
\end{tabular}

Fonte: os autores; imagem gerada no Excel.

O arquivo .xlsx deve conter cada estímulo que será apresentado, as condições de apresentação e a resposta correta. Outros parâmetros, como a cor específica de apresentação e outras variáveis, se for o caso, devem ser acrescentados nessa planilha, para viabilizar a extração dos dados de acordo com as variáveis de cada estudo.

Após o download e instalação do $P$ sychoPy ${ }^{2}$, o programa deve ser aberto e os passos abaixo devem ser seguidos para facilitar a replicabilidade do experimento:

a) Em View, selecione Go to Builder View;

b) No painel Flow, clique com o botão direito do mouse sobre Trial e remova-o. Em Insert routine, clique em new, digite Instructions e clique em $O K$. Por fim, clique na linha do fluxograma. Você terá criado sua primeira rotina.

\footnotetext{
${ }^{2} \mathrm{O}$ programa é instalado em língua inglesa. Segundo o manual, é possível configurar a língua em que os menus e outros comandos são exibidos (no ambiente do Builder, deve-se clicar em "preferences" e indicar "locale"). Todavia, não conseguimos realizar a referida configuração para a língua portuguesa. Por essa razão, os nomes, comandos e funções aqui reproduzidos sempre aparecem em língua inglesa.
} 
c) Clique na rotina Instructions. No painel Components, clique em Stimuli e selecione (Text). Uma nova janela aparecerá. Nessa janela, substitua o texto atual pelo texto de instruções do teste, que pode ser o seguinte: "A sua tarefa é focar na cor das palavras. Quando você vê a palavra escrita em verde, pressione a tecla verde (tecla j). Quando você vê a palavra escrita em azul, pressione a tecla azul (tecla 1). Quando você vê a palavra escrita em rosa, pressione a tecla rosa (tecla i). Tente responder da forma mais rápida e correta possível. Primeiramente, você fará um treino. Pressione espaço para continuar." Depois disso, defina o tempo de exibição desse texto apagando o campo Stop duration. Clique em $O K$ e você terá criado um componente da rotina Instructions.

d) No painel Components, clique em Responses e selecione (Keyboard). Uma nova janela aparecerá. Nessa janela, apague o campo Stop duration. Marque a opção force end of routine e digite 'space' no campo Allowed keys \$. Clique em Store e selecione nothing para que o programa não memorize as medidas da tela de instrução. Clique em $O K$ e você terá criado mais um componente da rotina Instructions. Neste caso, o componente Keyboard se refere ao comando que deve ser dado ao computador pelo participante para que o texto de instruções deixe de ser exibido e a execução do teste prossiga.

e) Salve o experimento na mesma pasta em que o arquivo Excel foi salvo. Para testar o experimento, clique em (Run). Uma nova janela abrirá e solicitará o nome do participante e o número da sessão. Você pode sair do experimento a qualquer momento ao apertar a tecla Esc.

f) No painel Flow, clique em Insert routine e escolha new. Atribua um nome a essa rotina (Fixation), clique em $O k$ e clique no ponto do fluxograma no qual você deseja que essa rotina apareça, ou seja, depois de Instructions.

g) Clique na rotina Fixation, em Stimuli e selecione 1 r. Uma nova janela aparecerá. Nessa janela, substitua o texto atual pelo operador + e selecione a opção set every repeat. Após, defina o tempo de exibição no campo Stop duration em 0.4 (sugestão: 400 milissegundos). Digite $\$[-1.000,-1.000,-1.000]$ (sugestão: cor branca) no campo Color ou clique com o botão direito para selecionar uma cor e clique em $O K$.

h) No painel Flow, clique em Insert routine e escolha new. Atribua um nome a essa rotina (sugestão: "Practice trials") e clique em um ponto do fluxograma após Fixation.

i) Na rotina Practice trials, clique em Stimuli e selecione $\mathfrak{T}$. Substitua o texto por \$word e selecione a opção set every repeat. Defina o campo Stop duration com 2.0. Em relação ao campo Color, digite \$colour e marque a opção set every repeat (\$colour remete ao conteúdo da coluna intitulada correct na planilha de dados). Clique em $O K$. 
j) Ainda na rotina Practice trials, clique em Responses e selecione $\square$. Defina os tempos de duração com Start time (s) em 0.1 e Stop duration (s) em 2.0. Marque a opção Force end of routine, digite 'j','l','i' no campo Allowed keys \$ e escolha a opção Set every repeat. Também marque a opção Store correct e digite \$correct no campo Correct answer (\$correct remete ao conteúdo da coluna intitulada correct na planilha de dados). Clique em $O K$.

k) Ainda na rotina Practice trials, insira um novo componente com o nome Feedback.

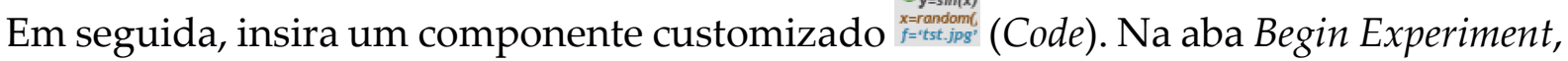
digite message $=$ ' '. Na aba Begin Routine, insira o seguinte código:

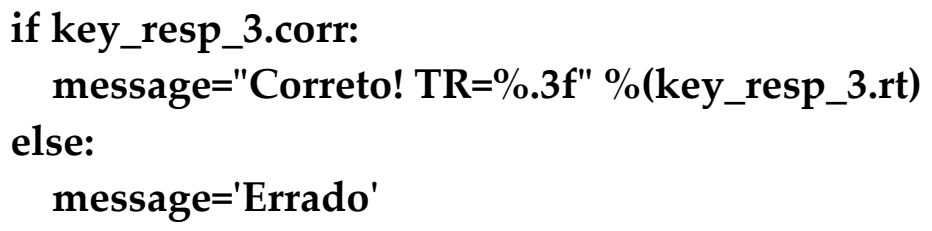

Key_resp_3 refere-se ao componente Responses > Keyboard utilizado para responder sobre a palavra/estímulo. Por isso, é necessário verificar o nome atribuído ao teclado na rotina anterior. Clique em $O K$.

1) Ainda na rotina Feedback, insira um componente de texto. Configure a duração (sugestão: 1 ou 2 segundos), a cor e as demais características de acordo com a rotina anterior. Em Text, digite \$message e marque Set every repeat. Clique em OK e execute o experimento clicando em 6 .

m) No painel Flow, clique em Insert loop e clique em um ponto do fluxograma antes da rotina Fixation e após a rotina Feedback. Uma nova janela abrirá. Selecione random em Loop type e marque a opção Is trials. No campo $n$ Reps $\$$ indique o número de repetições para cada trial $^{3}$ do arquivo de condições (sugestão: 3). Por fim, no campo Conditions, busque o arquivo de condições stroop.xlsx clicando em Browse. Clique em $O K$.

As configurações de fonte, cor e tamanho dos elementos textuais são facilmente modificáveis dentro do próprio componente Text. A disposição dos elementos textuais e de outros elementos gráficos no ambiente de experimentação também pode ser

\footnotetext{
${ }^{3}$ Trial refere-se a cada linha do arquivo de condições, ou seja, diz respeito a cada condição experimental a que o participante será submetido.
} 
manipulada por meio de coordenadas atribuídas aos referidos componentes. A utilização da cor branca no fundo da tela facilita a visualização do experimento e impede, na maioria dos casos, os efeitos indesejados de reflexos. A atribuição da cor branca ao ambiente de experimentação pode ser facilmente configurada no menu principal do módulo Builder (Experiment settings > Screen > Color: $\$[1.000,1.000,1.000]$ ). Por fim, a ordem de apresentação dos estímulos (por exemplo, aleatória ou sequencial) pode ser modificada dentro do loop.

Apresentamos somente o roteiro da sessão de treinamento, os blocos do teste seguem a mesma estrutura e devem ser separados por uma nova rotina denominada Pausa (cf. figura 2).

\subsection{Execução}

O teste Stroop foi aplicado a três grupos de participantes. Os grupos foram compostos considerando o perfil linguístico dos participantes. O grupo denominado GHA ( $\mathrm{n}=28)$ foi composto por multilíngues falantes de hunsriqueano riograndense (HR), língua minoritária brasileira de origem alemã (cf. ALTENHOFEN, 1996), português brasileiro (PB) (ambas as línguas foram adquiridas pelos participantes durante a infância), alemão standard (AS) e outras línguas, também adquiridas em contexto escolar. No grupo GH $(n=29)$, havia participantes falantes de HR e PB, também adquiridos precocemente, bem como de outras línguas. O grupo-controle, ou seja, o grupo GA $(n=28)$ foi constituído de falantes de $\mathrm{PB}$, AS e outras línguas. Os integrantes do grupo-controle não possuíam conhecimento de qualquer língua minoritária de origem alemã. Para todos os grupos, conhecimentos de outras línguas, sobretudo de inglês e espanhol configuraram o multilinguismo (cf. DE ANGELIS, 2007; HERDINA; JESSNER, 2004).

Por meio de um questionário (cf. LIMBERGER, 2018), baseado em Scholl e Finger (2013), constatamos que os voluntários $(n=85)$ são adultos jovens (média de 
idade $=27,4$; desvio padrão $=6,3)$, sendo a maioria do sexo feminino $(=68)$. Todos os participantes possuíam nível de escolaridade superior em curso ou completo. Os participantes declararam não possuir problemas de audição e possuir visão normal ou corrigida. Nenhum dos participantes relatou ter algum transtorno de linguagem ou aprendizagem. Pode-se dizer que os perfis dos grupos são similares entre si, com pouca variabilidade no que concerne às médias dos grupos. Compuseram a amostra participantes com ocupações/profissões variadas.

Aos 85 participantes que se enquadraram nos critérios de inclusão foi aplicado o experimento, conforme reportado em Limberger (2018). Para a execução da tarefa, foi utilizado um notebook HP Pavilion 14'. A coleta de dados ocorreu entre abril e agosto de 2017 e o local variou de acordo com o desejo e a disponibilidade dos participantes, contanto que a coleta ocorresse em alguma sala iluminada e silenciosa.

\subsection{Resultados}

Os resultados do teste Stroop fornecem um índice da capacidade de inibição de estímulos linguísticos durante o processamento da leitura que consiste na subtração da média de $R T$ (reaction time) para itens incongruentes e da média do $R T$ para itens congruentes. Parte do arquivo .csv gerado pelo PsychoPy e visualizado no Excel foi reproduzido a seguir (cf. Figura 5). 
Figura 5 - Arquivo com extensão .csv, visualizado no Excel.

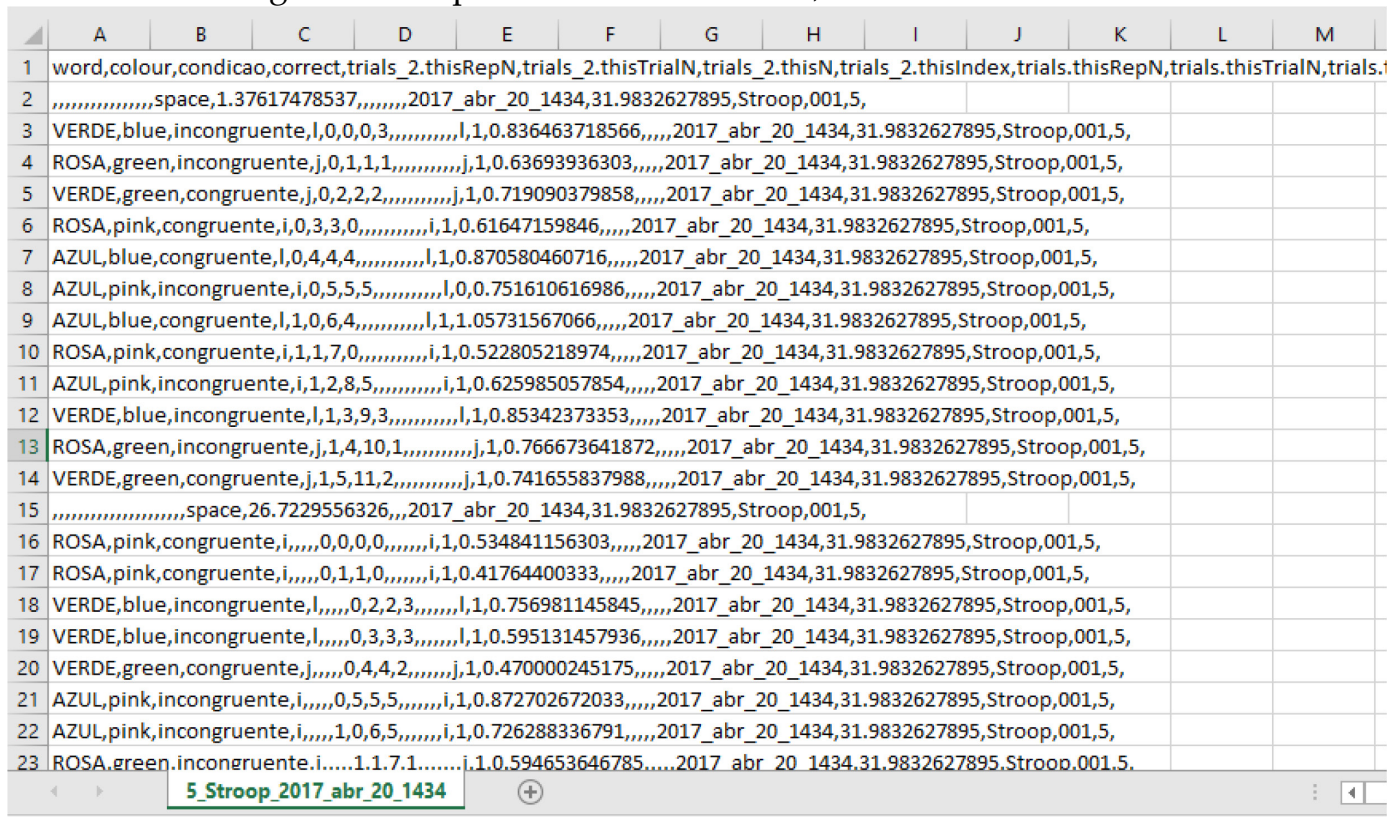

Fonte: os autores; imagem gerada no Excel.

No Excel, a função “Texto para colunas" pode ser usada para facilitar a visualização dos resultados. Para tanto, deve-se selecionar a primeira coluna, clicar em "Dados", então em “Texto para colunas" e marcar a opção "separar o texto por vírgulas".

O PsychoPy gera uma planilha de resultados para cada participante, o que é uma das suas desvantagens, pois a aplicação do teste entre inúmeros participantes resulta em uma quantidade relativamente grande de arquivos que, em algum momento, deverão ter seus dados agregados em uma única planilha para que o devido tratamento estatístico seja levado a cabo. Há recursos que otimizam esse processo de fusão entre diferentes arquivos, como, por exemplo, com o auxílio de um arquivo com extensão .bat, que pode ser definido como um conjunto de comandos executados em lote.

O programa mensura cada acerto por 1 e cada erro por 0. Assim, é possível fazer uma média de 0 a 1 ou, ainda, calcular a porcentagem por meio de uma regra de três simples. Finalmente, o RT deve ser transformado em milissegundos. 
No teste Stroop, os participantes tiveram altos níveis de acurácia, pois o experimento não apresentava níveis muito grandes de dificuldade. Por isso, foi observado um efeito-teto nessa variável. Na Tabela 1 a seguir, apresentamos as médias e os desvios-padrão de $R T$, os quais foram gerados no software SPSS 119.0 (IBM CORP., 2010).

Tabela 1 - Escores dos três grupos no Efeito Stroop (ms) e desvios padrão (entre parênteses).

\begin{tabular}{cccc} 
& GHA & GH & GA \\
\hline $\begin{array}{c}\text { Efeito Stroop } \\
\text { (DP) }\end{array}$ & $59(254)$ & $54(230)$ & $66(346)$ \\
\hline
\end{tabular}

Uma ANOVA unifatorial indicou diferença não significativa em relação ao efeito Stroop. Desse modo, na análise dos resultados dos testes de processamento da leitura em multilíngues, a explicação para eventuais diferenças entre o desempenho dos grupos não estaria atrelada ao controle inibitório (LIMBERGER, 2018). Esse controle foi importante porque é notório que o controle inibitório e as funções executivas de modo geral têm uma relação direta com o processamento bilíngue/multilíngue (ANTONIOU et al., 2016; BIALYSTOK et al., 2009; KRAMER; MOTA, 2015; LIMBERGER, 2014).

\section{Experimento 2: Tarefa de decisão lexical}

A tarefa de decisão lexical visa avaliar o acesso lexical, que pode ser definido, basicamente, pelo processo de recuperar informações semânticas e gramaticais por meio da forma ortográfica ou fonológica (DIJKSTRA, 2005). Estudos com bilíngues e multilíngues têm sugerido que o acesso lexical é não seletivo (cf. DE BOT, 2004; DIJKSTRA; GRAINGER; VAN HEUVEN, 1999). Portanto, a tendência é de que as representações das duas ou três línguas sejam ativadas e entrem em competição durante a seleção lexical, ocorrendo uma busca paralela em todos os subsistemas e, 
consequentemente, influências interlinguísticas. Em tese, palavras cognatas são processadas de modo mais rápido, o que é resultante do compartilhamento de representações entre as duas línguas (DIJKSTRA, 2005).

A tarefa de decisão lexical apresentada neste estudo contempla as interações entre as línguas portuguesa e hunsriqueana. Entretanto, diferentemente do teste Stroop, a tarefa de decisão lexical foi testada com um único grupo durante o estudo piloto reportado por Limberger (2018). Esse estudo foi conduzido a fim de garantir que um eventual efeito cognato não ocorreria por fatores não controlados relativos aos estímulos. Assim sendo, participantes que não possuem conhecimento da língua-alvo não deveriam ser influenciados pela sobreposição de representações no léxico mental.

Os 90 estímulos selecionados para essa tarefa foram palavras cognatas (PB-HR), que são empréstimos ou estrangeirismos que o HR tomou do PB (cf. SCHAUMLOEFFEL, 2003; ALTENHOFEN et al., 2007). Além disso, selecionamos palavras em português (condição PB) que não são emprestadas. As pseudopalavras (PseudoPB) foram retiradas de Salles e Parente (2002) e Rodrigues e Salles (2013), que as validaram. As pseudopalavras foram pareadas entre as condições considerando extensão, número de letras, sílabas e encontros consonantais. No caso das palavras reais, foi possível controlar também a frequência e o número de vizinhos ortográficos. O experimento foi composto, portanto, de três condições constituídas por 30 estímulos cada.

Primeiramente, os participantes realizaram um treino com seis palavras, que pode ser repetido. Logo em seguida, eles fizeram o teste com 90 estímulos. Conforme representado na figura 6, cada palavra aparecia durante 2000 milissegundos (ms), seguida de um ponto de fixação (400 ms). Para determinar se houve um efeito dos cognatos, calcula-se a diferença entre os resultados da leitura dos itens não-cognatos e dos itens cognatos. 
Figura 6 - Representação esquemática da Tarefa de decisão lexical.

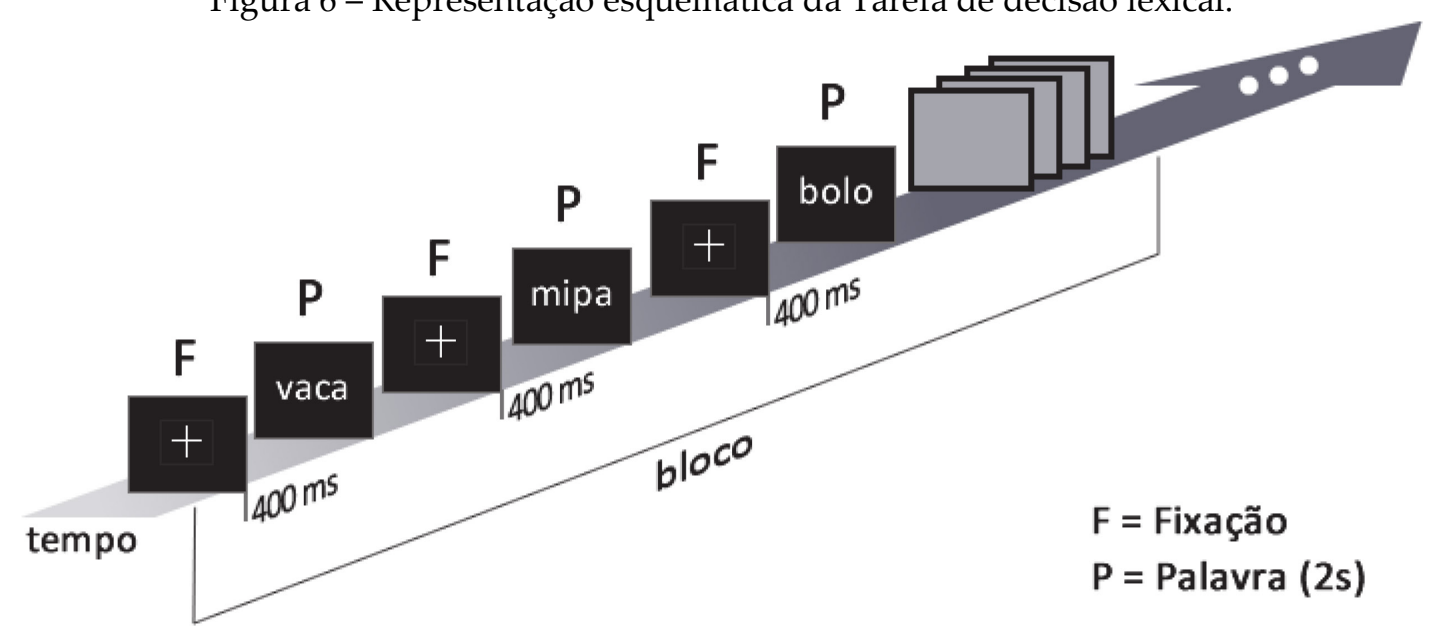

Fonte: os autores; imagem gerada no software Adobe Fireworks.

Todos os estímulos foram apresentados no centro da tela com cor de fundo preta e na fonte Arial (tamanho 18) na cor branca. Os participantes liam uma palavra apresentada na tela do computador uma por vez e respondiam à pergunta "Essa palavra existe? SIM ou NÃO". Em caso positivo, eles pressionavam a tecla "S" do computador devidamente sinalizada com uma etiqueta na cor verde; em caso negativo, a tecla "L" sinalizada com uma etiqueta vermelha ${ }^{4}$.

\subsection{Construção do experimento no PsychoPy}

Fundamental para o funcionamento do experimento a ser construído no PsychoPy, é a planilha de condições criada no Excel (Figura 7), que reproduzimos a seguir. Reitera-se que cada linha corresponde a um trial ou condição experimental.

\footnotetext{
${ }^{4}$ Essas teclas foram escolhidas pelos pesquisadores devido a sua localização no teclado do notebook, ou seja, bem distantes entre si, para não haver confusão.
} 
Figura 7 - Arquivo de condições da Tarefa de decisão lexical.

\begin{tabular}{|c|c|c|c|}
\hline$\Delta$ & $\mathrm{A}$ & B & C \\
\hline 1 & word & correct & condition \\
\hline 2 & abacaxi & $s$ & PB-HR \\
\hline 3 & abelha & s & PB \\
\hline 4 & alanare & I & PseudoPB \\
\hline 5 & arfa & I & PseudoPB \\
\hline 6 & arroz & 5 & PB \\
\hline 7 & bala & 5 & PB-HR \\
\hline 8 & balcão & $s$ & PB-HR \\
\hline 9 & barba & $s$ & PB \\
\hline 10 & barranco & 5 & PB-HR \\
\hline 11 & bico & $s$ & PB-HR \\
\hline 12 & bife & $s$ & PB-HR \\
\hline 13 & bilhete & $s$ & PB-HR \\
\hline 14 & bolo & s & PB-HR \\
\hline 15 & borboleta & $s$ & $\mathrm{~PB}$ \\
\hline
\end{tabular}

Fonte: os autores; imagem gerada no Excel.

Não há muitas diferenças entre as estruturas da tarefa de decisão lexical e do teste Stroop. Por essa razão, as instruções apresentadas na seção 3.1 podem ser seguidas. Ainda em relação aos procedimentos de montagem da tarefa de decisão lexical, destacamos que os tempos de apresentação dos estímulos podem variar de acordo com os níveis linguísticos investigados e o objetivo da pesquisa. Além disso, evidentemente, as teclas de resposta e as instruções deverão ser diferentes. Como sugestão para as instruções da tarefa de decisão lexical, temos: "Nesta tarefa, você lerá a palavra exibida na tela uma por vez. Quando a palavra for uma palavra real, aperte a tecla "s" (tecla verde). Quando a palavra não existir, aperte a tecla "1" (tecla vermelha). Atente para sempre responder da forma mais correta e rápida possível. Por gentileza, tente responder dentro de 2 segundos. Primeiramente, você fará um treino com oito palavras. Clique no espaço para começar a tarefa."

\subsection{Execução}

O experimento foi aplicado a 21 adultos (16 mulheres e 5 homens) com idades entre 18 e 50 anos (média = 25,5 anos; DP = 8,5). Todos eram estudantes da PUCRS 
(média dos anos de escolaridade =15,5 anos; $\mathrm{DP}=3,5$ ). Nenhum dos participantes tem contato com qualquer língua minoritária de origem alemã, e todos se consideraram bilíngues, falantes de PB (L1) e de inglês $(n=17)$ ou espanhol $(n=4)$ como L2. Os participantes declararam apresentar condições de audição e visão perfeitas e nenhum transtorno neuropsicológico. A coleta de dados ocorreu em março de 2017 numa sala silenciosa na Faculdade de Letras da PUCRS.

\subsection{Resultados}

A visualização dos resultados não difere muito daquela apresentada anteriormente na Figura 5. Por isso, é possível adotar os mesmos procedimentos descritos para o teste Stroop.

Uma ANOVA unifatorial revelou diferenças significativas nas duas variáveis dependentes mensuradas pelo PsychoPy, a saber, acurácia $(F(2,19)=20,95, p<0,001)$ e $\operatorname{TR}(F(2,19)=108,740, p<0,001)$. Comparações post hoc com o teste de Bonferroni indicaram que, na leitura de pseudopalavras, os participantes apresentaram índices de acurácia significativamente menores $(p<0,001)$ em comparação à leitura de palavras cognatas (condição PB-HR) e não-cognatas (condição PB).

Uma vez que o PsychoPy também possibilita verificar os resultados de cada estímulo individualmente, é possível identificar estímulos que, por não serem adequados, não são processados com muita acurácia. Na tarefa aqui discutida, foi necessário excluir duas pseudopalavras que estavam sendo processadas como palavras, o que foi possível verificar pelos baixos índices de acurácia. 
Tabela 2 - Médias de tempo de resposta (ms) e de acurácia (porcentagem) e desvios padrão (entre parênteses) dos participantes do estudo piloto da Tarefa de decisão lexical em PB.

\begin{tabular}{ccc} 
Condição & TR (DP) & $\begin{array}{c}\text { Acurácia } \\
\text { (DP) }\end{array}$ \\
\hline PB-HR & $663(173)$ & $98(27)$ \\
PB & $646(175)$ & $98(12)$ \\
PseudoPB & $810(284)$ & $92(27)$ \\
Efeito PB-HR sobre PB & -17 & 0 \\
\hline
\end{tabular}

Os resultados reproduzidos na Tabela 2 sugerem que os participantes tiveram mais dificuldades em reconhecer pseudopalavras do que palavras, o que é um resultado recorrente nos estudos que se utilizam da tarefa de decisão lexical. A ausência de diferenças entre a leitura das palavras cognatas e não-cognatas sugere que os estímulos foram controlados e os participantes não sofreram qualquer efeito que diferencie o desempenho em cada tipo de palavra, o que era desejado.

\section{Discussão}

O PsychoPy se mostrou plenamente adequado à investigação sobre o processamento da leitura. A nossa experiência tem sido positiva com o programa; também por isso, decidimos compartilhá-la. Como já sinalizado anteriormente, o programa possui facilidades e dificuldades, as quais detalharemos nesta seção.

No que tange à investigação do processamento da leitura, o programa possibilita investigar a leitura em seus diversos níveis e em relação a construtos cognitivos. Os experimentos apresentados neste artigo contemplam o processamento lexical. Para o teste Stroop, é essencial a apresentação das palavras em diferentes cores, 
o que é um recurso disponível no PsychoPy por meio de nomes específicos conforme a linguagem de programação HTML ${ }^{5}$.

Apesar de ser sensível a diacríticos, é possível apresentar como estímulo textual qualquer caractere. Isso abrange qualquer sistema de escrita e qualquer diacrítico (por exemplo, como os acentos, o til e o cedilha em português). Além disso, nos experimentos exemplificados, trabalhamos com palavras isoladas, mas não há limite de palavras para serem apresentadas pelo PsychoPy. Além disso, o programa não exige muito conhecimento sobre programação, e há vários tutoriais e manuais disponíveis na rede. Encontramos, inclusive, um tutorial em português, produzido pela professora Mahayana Godoy da Universidade Federal do Rio Grande do Norte'.

Outras facilidades encontradas por meio da programação e execução das tarefas se relacionam às variáveis sob investigação. As variáveis dependentes mais frequentemente investigadas em estudos comportamentais são o tempo de resposta (RT) e a acurácia. O PsychoPy mensura essas variáveis com precisão. Apesar disso, precisamos estar cientes de que esses dados nem sempre são reais, pois o participante pode ter pressionado a tecla por impulso, por exemplo. Neste caso, é possível, entre outros, configurar o experimento para que o tempo de resposta não possa ser registrado imediatamente. Além disso, pode-se estabelecer que as respostas fornecidas antes de determinado tempo de resposta serão desconsideradas para fins de computação dos dados. Chamamos a atenção para o fato de que o $R T$ não é o tempo de leitura, mas sim de resposta, porque há o tempo de apertar a tecla. Finalmente, Plant (2016) e Sampaio (2017) salientam que limitações de hardware podem interferir na captura precisa destes dados pelo PsychoPy. De acordo com Peirce (2009), a precisão temporal pode ser afetada principalmente em razão da taxa de atualização (frame rate)

\footnotetext{
${ }^{5}$ Lista dos nomes disponível em: https://www.w3schools.com/Colors/colors_names.asp. Acesso em: 29 ago. 2018.

${ }^{6}$ Disponível em: https://www.youtube.com/watch?v=W8cpnARvtNw\&t=14s. Acesso em: 30 ago. 2018.
} 
dos monitores e do tempo de latência dos teclados utilizados nos experimentos. Os autores do programa salientam que resultados muito precisos podem ser obtidos através da apresentação de estímulos utilizando a taxa de atualização do monitor em vez do tempo em milissegundos, bem como da utilização de um button box para registro das respostas.

Por ser um programa de código aberto, o PsychoPy está em constante atualização, o que pode ser constatado na página de download do programa․ Por essa razão, o programa está sendo constantemente aperfeiçoado. Há também fóruns online que auxiliam nessa tarefa e a solucionar os problemas. Esse recurso foi crucial para o desenvolvimento dos experimentos aqui apresentados, principalmente no início da tarefa. Um exemplo da contribuição fundamental dos fóruns foi o acesso aos códigos utilizados no componente feedback.

Em suma, concordamos com Sampaio (2017), que recomenda PsychoPy para aqueles que estão iniciando a sua pesquisa psicolinguística. Além dos motivos já elencados neste artigo, o autor acrescenta a possibilidade de trocar informações e códigos com pesquisadores do mundo inteiro, porque o software é bastante popular no mundo. Além disso, Sampaio relata a sua experiência com aulas de psicolinguística na graduação, em que tem tido sucesso o trabalho com o PsychoPy. Ao criar experimentos no programa, os seus alunos perdem o medo que têm devido à falta de conhecimentos em programação e podem ter uma experiência hands-on já em sala de aula. Esse relato corrobora as impressões que tivemos durante as oficinas que ministramos para alunos de Letras (graduação e pós-graduação), nas quais os participantes conseguiam compreender o funcionamento do programa e criar um experimento em poucas horas.

7 Disponível em https:/github.com/psychopy/psychopy/releases. Para executar o programa, aconselhamos a primeira versão com a extensão .exe. Acesso em: 30 ago. 2018. 
Como o próprio desenvolvedor do programa adverte, o PsychoPy não é bugfree $^{8}$. É provável, portanto, tal como nossa experiência comprovou, que surgirão problemas para rodar os experimentos, principalmente quando começamos a trabalhar com o programa. Uma das dificuldades recorrentemente observadas é quando salvamos o experimento na área de trabalho do computador. Isso ocorre porque o caminho que o Python precisa ler não pode conter qualquer diacrítico, o que é o caso do acento agudo da palavra 'área'. Então, é aconselhável utilizar outra pasta para salvar o experimento.

Uma das dificuldades enfrentadas foi o trabalho com as planilhas que são geradas com extensão .csv, pois é necessário separar as colunas quando se trata os dados no SPSS. Essa tarefa não é de difícil resolução, conforme explicado anteriormente (subseção 3.3). Outro problema foi agregar as colunas; para tanto, foi necessário desenvolver um comando (Arquivo em Lotes do Windows, .bat) para facilitar o trabalho. Depois de agregar, é necessário deletar os cabeçalhos e as informações dos participantes, de modo que restem somente os dados.

\section{Considerações finais}

Este artigo teve como principal objetivo analisar o uso do programa PsychoPy na investigação do processamento de leitura no que tange ao desenvolvimento, execução e tratamento dos resultados de dois experimentos psicolinguísticos. Esperamos mostrar aos pesquisadores da área e a outros interessados as principais vantagens e desvantagens do programa.

Dentre as desvantagens, há o fato de que é necessário conhecer minimamente a linguagem de programação Python para resolver alguns bugs e escrever determinados códigos. Em outras palavras, um conhecimento básico da sintaxe em Python se faz

\footnotetext{
${ }^{8}$ No original: "Running on all possible hardware and all platforms is a big ask." Disponível em: http://www.psychopy.org/troubleshooting.html. Acesso em: 29 ago. 2018.
} 
necessária para solucionar problemas em alguns casos. Ademais, destacamos o modo como são apresentados os resultados. Tivemos dificuldades para organizar e agregar as planilhas de Excel, pois o programa gera para cada participante uma planilha separada. Sugestões para solucionar esse problema foram apresentadas ao longo deste artigo.

Nos estudos que revisamos na introdução, foram investigados sobretudo aspectos sintáticos, pragmáticos e lexicais com a apresentação de estímulos visuais. Os estudos que utilizam estímulos sonoros frequentemente enfrentam dificuldades em relação às bibliotecas de áudio utilizadas pelo PsychoPy, constituindo, por vezes, uma dificuldade a mais na construção dos experimentos ${ }^{9}$.

Quanto às vantagens do uso do PsychoPy, destacam-se a potencialidade de uso do PsychoPy no contexto da pesquisa linguística brasileira, uma vez que o programa é livre e gratuito, de fácil aprendizagem e implementação, corroborados pela aplicação dos testes Stroop e de decisão lexical apresentados no presente artigo.

Destaca-se também que o PsychoPy torna possível a realização de uma gama de investigações no campo dos estudos comportamentais, pois o programa viabiliza a concretização de experimentos linguísticos com interface nas ciências cognitivas, na neurociência e na psicologia. Biasibetti (2018), a exemplo, construiu e aplicou o teste de associação implícita - um teste amplamente utilizado na área de Psicologia Social no PsychoPy para investigar a percepção sociofonética das fricativas sibilantes em coda silábica no português brasileiro.

Salienta-se também que para criar experimentos relativamente simples, o ambiente Builder viabiliza a construção desses experimentos de modo direto e intuitivo sem a necessidade de programar em Python. Com uma interface convidativa, o Builder

\footnotetext{
${ }^{9} \mathrm{O}$ segundo autor deste estudo enfrentou tal dificuldade nas etapas iniciais do desenvolvimento de testes de percepção fonética.
} 
foi utilizado para a construção dos experimentos Stroop e de decisão lexical apresentados no presente artigo.

Por fim, quaisquer erros ou dificuldades encontradas durante a instalação e utilização do programa podem ser facilmente sanadas através de fóruns on-line continuamente atualizados por uma comunidade de programadores e pesquisadores devotados à propagação de softwares livres e gratuitos que permitem a ampliação do conhecimento científico.

Assim posto, este artigo é um convite e um encorajamento para investigarmos o processamento, a percepção e a produção das diversas línguas e variedades linguísticas verificadas no Brasil, em diferentes populações e em diferentes modalidades e níveis linguísticos através da experimentação viabilizada pela construção e aplicação de testes através do programa PsychoPy.

\section{Agradecimentos}

Agradecemos ao CNPq, que concedeu bolsa de doutorado aos autores deste artigo, e aos pareceristas que contribuíram para o aperfeiçoamento deste trabalho.

\section{Referências}

ALTENHOFEN, C. V. Hunsrückisch in Rio Grande do Sul: Ein Beitrag zur Beschreibung einer deutschbrasilianischen Dialektvarietät im Kontakt mit dem Portugiesischen. Stuttgart: Steiner, 1996.

ALTENHOFEN, C. V. et al. Fundamentos para a escrita do Hunsrückisch falado no Brasil. Revista Contingentia, v. 2, n. 51, p. 73-87, 2007.

ANTONIOU, K. et al. The effect of childhood bilectalism and multilingualism on executive control. Cognition, v. 149, p. 18-30, 2016. DOI https://doi.org/10.1016/j.cognition.2015.12.002.

BIALYSTOK, E. et al. Bilingual Minds. Psychological Science in the Public Interest, v. 10, n. 3, p. 89-129, 2009. DOI https://doi.org/10.1177/1529100610387084. 
BIASIBETTI, A. P. C. S. Produção e percepção das fricativas sibilantes em Porto Alegre/RS e Florianópolis/SC. 2018. Tese (Doutorado em Letras). Pontifícia Universidade Católica do Rio Grande do Sul, 2018.

DE ANGELIS, G. Third or Additional Language Acquisition. Clevedon, UK: Multilingual Matters, 2007. DOI https://doi.org/10.21832/9781847690050

DE BOT, K. The Multilingual Lexicon: Modelling Selection and Control. International Journal of Multilingualism, v. 1, n. 1, p. 17-32, 2004. DOI https://doi.org/10.1080/14790710408668176.

DIJKSTRA, T. Bilingual visual word recognition and lexical access. In: KROLL, J.; DE GROOT, A. M. B. (ed.). Handbook of bilingualism: Psycholinguistic Approaches. Oxford: Oxford University Press, 2005. p. 179-201.

DIJKSTRA, T.; GRAINGER, J.; VAN HEUVEN, W. J. B. Recognition of Cognates and Interlingual Homographs: The Neglected Role of Phonology. Journal of Memory and Language, v. 41, n. 4, p. 496-518, 1999. DOI https://doi.org/10.1006/jmla.1999.2654

GODOY, M. C.; VIEIRA, C. B.; ANDRADE, G. L.; SOUZA FILHO, N. de; NUNES, M. A. O papel do conhecimento de eventos no processamento de sentenças isoladas. Revista Letrônica, v. 10, n. 2, p. 538-554, 2017. DOI https://doi.org/10.15448/19844301.2017.2.26408

GOMES, R. C. da C. Processamento de dativos por crianças bilíngues portuguêsinglês. 2017. Monografia. Universidade Federal da Paraíba, 2017.

HERDINA, P.; JESSNER, U. A Dynamic Model of Multilingualism: Perspectives of Change in Psycholinguistics. Clevedon/Buffalo/Toronto/Sydney: Multilingual Matters, 2002. DOI https://doi.org/10.21832/9781853595547

IBM CORP. Released 2010. IBM SPSS Statistics for Windows. New York: IBM Corp, 2010.

KRAMER, R.; MOTA, M. B. Effects of bilingualism on inhibitory control and working memory: a study with early and late bilinguals. Gragoatá, v. 38, p. 309-331, 2015.

LIMBERGER, B. K. Processamento da leitura e suas bases neurais: um estudo sobre o hunsriqueano. 2018. 269 p. Tese (Doutorado em Letras/Linguística) - Programa de 
Pós-graduação em Letras, Pontifícia Universidade Católica do Rio Grande do Sul, Porto Alegre, 2018.

LIMBERGER, B. K. O desempenho de bilíngues e multilíngues em tarefas de controle inibitório e compreensão auditiva. 2014. 135 f. Dissertação (Mestrado em Letras) - Programa de Pós-Graduação em Letras, Pontifícia Universidade Católica do Rio Grande do Sul, Porto Alegre, 2014. DOI https://doi.org/10.13140/RG.2.1.4437.4645

MIYAKE, A. et al. The Unity and Diversity of Executive Functions and Their Contributions to Complex "Frontal Lobe" Tasks: A Latent Variable Analysis. Cognitive Psychology, v. 41, n. 1, p. 49-100, 2000. DOI https://doi.org/10.1006/cogp.1999.0734

Neurobehavioral Systems Inc. Presentation. Disponível em: https://www.neurobs.com/

OLIVEIRA, T. L. de. Língua e percepção: o processamento dos clíticos com referência ao interlocutor no Português Brasileiro. Tese (Doutorado em Letras). Universidade Federal do Rio de Janeiro - UFRJ, 2018.

PEIRCE, J. W. PsychoPy - Psychophysics software in Python. Journal of Neuroscience $\begin{array}{lllllll}\text { Methods, } & \text { v. } 162, & \text { n. } 1-2, & \text { p. } & 8-13, & \text { DOI }\end{array}$ https://doi.org/10.1016/j.jneumeth.2006.11.017

PEIRCE, J. W. Generating stimuli for neuroscience using PsychoPy. Frontiers in Neuroinformatics, v. 2, p. 1-10, 2009. DOI https://doi.org/10.3389/neuro.11.010.2008

PEIRCE, J. W. PsychoPy - Psychology software for Python Release 1.90.2. Jun. 01, 2018.

PLANT, R. R. A reminder on millisecond timing accuracy and potential replication failure in computer-based psychology experiments: an open letter. Behavior Research Methods, v. 48, n. 1, p. 408-411, 2016. DOI https://doi.org/10.3758/s13428-015-0577-0

Psychology Software Tools, Inc. E-Prime 3.0, 2016. Disponível em: http://www.pstnet.com. Acesso em: 28 ago. 2018.

ROCHA, B. N. R. de M; RASO, T. A interação entre pistas prosódicas e pragmáticas para a interpretação ilocucionária de um enunciado. Anais do VI Colóquio Brasileiro de Prosódia da Fala, v. 4, p. 99-102, 2017. 
RODRIGUES, J. C.; SALLES, J. F. Tarefa de escrita de palavras/pseudopalavras para adultos: abordagem da neuropsicologia cognitiva. Letras de Hoje, v. 48, n. 1, p. 50-58, 2013.

SALLES, J. F.; PARENTE, M. A. M. P. Relação entre os processos cognitivos envolvidos na leitura de palavras e as habilidades de consciência fonológica em escolares. Prófono - Revista de Atualização Científica, v. 14, n. 2, p. 175-186, 2002.

SAMPAIO, T. O. da M. A escolha de software e hardware na psicolinguística: revisão e opinião. Revista de Estudos da Linguagem, v. 25, n. 3, 2017. DOI https://doi.org/10.17851/2237-2083.11283

SANTOS, T. de P. B. The influence of reading while listening to narratives on comprehension, spoken word recognition and lexical memory of EFL Brazilian learners. 2016. Dissertação (Mestrado em Letras). Universidade Federal de Santa Catarina, 2016.

SCHAUMLOEFFEL, M. A. Estudo da Interferência do Português da Variedade Dialetal Hunsrück Falada em Boa Vista do Herval. 2003. 136 f. Dissertação (Mestrado em Letras) - Programa de Pós-graduação em Letras, Universidade Federal do Paraná, Curitiba, 2003.

SCHOLL, A. P.; FINGER, I. Elaboração de um questionário de histórico de linguagem para pesquisas com bilíngues. Nonada Letras em Revista, v. 2, n. 21, p. 1-17, 2013.

SOUZA FILHO, N.; SILVA, G. L.; RODRIGUES, V. W.; ARAÚJO, F. N.; GODOY, M. C. Relações de coerência e resolução de anáforas. Linguística Rio, v. 3, n. 1, 2017.

STROOP, R. Studies of interference in serial verbal reactions. Journal of Experimental Psychology, v. 18, p. 643-662, 1935. DOI https://doi.org/10.1037/h0054651 